\title{
Metodología para la Estimación de Incertidumbres Asociadas a Concentraciones de Sólidos Suspendidos Totales Mediante Métodos de Generación Aleatoria
}

\author{
Andrés E. Torres-Abello ${ }^{1}$
}

\section{Resumen}

Este trabajo consistió en definir una metodología para estimar las incertidumbres asociadas a concentraciones de Sólidos Suspendidos Totales (SST) ligados a la fase de análisis de laboratorio, contemplando no solamente la precisión de los instrumentos de laboratorio sino también el submuestreo y la manipulación de muestras y aparatos de laboratorio. La metodología propuesta tiene en cuenta el cálculo de la incertidumbre de réplicas por medio de métodos analíticos, la comparación entre los resultados de réplicas por medio de pruebas t y el cálculo de la incertidumbre compuesta por medio del método de Monte Carlo. A manera de ejemplo, la metodología desarrollada se aplica a datos de concentración de SST obtenidos en laboratorio. Dichos datos corresponden a muestras reales con bajas, medianas y elevadas concentraciones de SST y con diferencias en las variabilidades submuestrales. Posteriormente se realizan comparaciones entre las incertidumbres obtenidas al aplicar el método propuesto y aquellas obtenidas teniendo en cuenta únicamente la precisión de los aparatos de medición utilizados en laboratorio. De estas comparaciones se pudo observar que se pueden presentar diferencias importantes tanto en los resultados de las incertidumbres asociadas a los resultados de concentraciones como a los valores de concentración asociados a las muestras.

1 Grupo de investigación Ciencia e Ingeniería del Agua y el Ambiente, Pontificia Universidad Javeriana, sede Bogotá, andres.torres@javeriana.edu.co 


\section{Palabras clave}

Sólidos Suspendidos Totales, incertidumbre compuesta, método de Monte Carlo, metrología, propagación de incertidumbres.

\section{Abstract}

In this work a methodology for the estimation of uncertainties associated to Total Suspended Solids Concentrations (TSS) concentrations obtained during laboratory analysis, that takes into account not only the precision of the laboratory instruments, but also subsampling, sample, and equipment manipulation, is proposed. The proposed methodology includes the computation of replicates uncertainties with analytical methods, the comparison between replicates through t-tests, and computation of uncertainties applying a Monte Carlo method. As an example, this methodology was applied to TSS concentration data obtained in laboratory. The data set used corresponds to real samples with low, medium, and high TSS concentrations and with different subsampling variabilities. Subsequently, comparisons between uncertainties obtained from the proposed methodology and those obtained when only equipment precision is taken into account were undertaken. From these comparisons it was observed that some important differences can exist between concentrations uncertainties as well as samples mean concentrations.

\section{Keywords}

Total Suspended Solids, composed uncertainty, Monte Carlo method, metrology, propagation of uncertainties. 


\section{INTRODUCCIÓN}

Entre los parámetros de calidad de aguas más utilizados se encuentran las concentraciones en Sólidos Suspendidos Totales (SST), Demanda Química de Oxígeno (DQO) y Demanda Bioquímica de Oxígeno a los 5 días $\left(\mathrm{DBO}_{5}\right)$. Tradicionalmente, estos resultados de calidad de aguas son obtenidos a partir de muestreos puntuales en campo, acoplados a análisis de laboratorio y se utilizan con frecuencia como una herramienta de gestión y de ayuda a la toma de decisiones para mejorar la calidad de los ecosistemas acuáticos mediante acciones estructurales (como sistemas de tratamiento al final del tubo y en la fuente, aliviaderos de sistemas de alcantarillado, entre otros) y no estructurales (como políticas de limpieza urbana, de gestión y mantenimiento de redes, entre otros). Adicionalmente, éstos se comparan a menudo con valores límites permisibles, utilizados por las autoridades ambientales como valores de control exigidos a los operadores de plantas de tratamiento públicas y privadas, sirven para identificar tendencias en la dinámica de las concentraciones de contaminantes, útiles en las dosificaciones y operación eficiente de las plantas de tratamiento, o se utilizan como datos de entrada en modelos de transporte contaminante (Ghestem \& Lachenal, 2008).

Debido a lo anterior, la calidad de los resultados de calidad de aguas debe evaluarse, con el fin de garantizar la pertinencia de las acciones propuestas (como la exigencia del pago de tasas retributivas por contaminación hídrica, concepción y construcción de sistemas de descontaminación, planeación de la operación de plantas de tratamiento, entre otros) o soportar el análisis crítico de los resultados arrojados por los modelos hidrodinámicos y los simuladores de calidad de aguas (Isukapalli \& Georgopoulos, 2001; Smith, 2002; Radwan \& Willems, 2008). Así como los reportes de calibración de equipos de laboratorio, el análisis de los procesos y procedimiento empleados, la revisión de los procedimientos utilizados para la calibración, los reportes del departamento de calidad de los laboratorios y los protocolos de trazabilidad, uno de los elementos para juzgar la calidad de un resultado de medición es su incertidumbre, y por lo tanto éste último se convierte en un indi- 
cador indispensable para interpretar de manera correcta un resultado (Taverniers et al., 2004a; 2004b).

En nuestro medio, para cada muestra de agua se realiza usualmente un único ensayo mediante el cual se estima el valor de cada parámetro de calidad de interés (Ministerio de Salud, 1984; Ministerio de Desarrollo Económico, 2000; Ministerio de la Protección Social, Ministerio de Ambiente Vivienda y Desarrollo Territorial, 2007). Ese único valor, el cual se reporte usualmente acompañado de la incertidumbre asociada a la precisión de los instrumentos de laboratorio utilizados, se utiliza como uno de los indicadores para calificar la calidad de dicho cuerpo de agua en el instante de la toma de muestra. Sin embargo, la incertidumbre de un resultado de calidad de aguas no depende únicamente de la precisión de los instrumentos de laboratorio. En efecto, la incertidumbre de medición está definida como un parámetro que caracteriza la dispersión de los valores que puede razonablemente atribuirse al mensurando, término definido por el Centro Español de Metrología, CEM (2008) como la magnitud sujeta a medición (JCGM, 2008).

En consecuencia, las incertidumbres relacionadas con el muestreo y submuestreo (muestreo realizado a partir de una muestra) tanto en campo como en laboratorio, así como el almacenamiento y la conservación de las muestras y la manipulación tanto de las muestras como de los aparatos constituyen una parte fundamental de la incertidumbre asociada a un resultado (Harmel et al., 2009). Para el caso de un único ensayo en laboratorio por muestra, la guía de EURACHEM/CITAC (2000) propone una metodología para la estimación de la incertidumbre en 5 etapas: (i) especificación del mesurando, donde se definen las etapas necesarias para la medición; (ii) identificación de las fuentes de incertidumbre; (iii) cálculo de las incertidumbres estándar asociadas a cada fuente de incertidumbre; (iv) cálculo de la incertidumbre estándar compuesta utilizando la ley de propagación de incertidumbres; y (v) cálculo de la incertidumbre ampliada, mediante un multiplicador, generalmente tomado como 2 , de manera a expresarla con un $95 \%$ de confianza.

Teniendo únicamente muestras puntuales obtenidas en campo para evaluar la calidad del agua, acopladas a un único ensayo en 
laboratorio por muestra, no es posible evaluar la incertidumbre de manera realista y por lo tanto no se tiene un criterio para emitir juicios sobre la calidad, fidelidad y validez del resultado obtenido. Con el fin de evaluar incertidumbres sobre los resultados de manera más acertada, es recomendable realizar repeticiones de los ensayos sobre la misma muestra, lo que se denominará en este artículo como réplicas. Esto se debe hacer mediante submuestreos. De esta manera se puede evaluar no solamente la incertidumbre de cada resultado, mediante una metodología similar a la propuesta por EURACHEM/CITAC (2000), sino que también es posible evaluar la dispersión entre los resultados obtenidos para una misma muestra.

Los métodos numéricos para evaluar la incertidumbre, como el método de Monte Carlo, se utilizan cuando no es posible o se hace muy difícil utilizar métodos analíticos (Poulter, 1998; Greenland, 2001; Smith, 2002). En el caso de que los resultados de cada repetición no tengan una dispersión elevada, la evaluación de la incertidumbre asociada al resultado característico de la muestra puede evaluarse mediante métodos analíticos, como la ley de propagación de incertidumbres, aplicada a la ecuación del valor promedio. Sin embargo, no existe una metodología establecida para la evaluación de la incertidumbre del resultado de la muestra teniendo en cuenta réplicas cuando existe una dispersión elevada entre los resultados asociados a dichas réplicas.

Este trabajo tiene por objetivo definir una metodología para estimar las incertidumbres asociadas a resultados de concentraciones de Sólidos Suspendidos Totales (SST) sobre muestras puntuales obtenidas en campo, contemplando no solamente la precisión de los instrumentos de laboratorio sino también el submuestreo y manipulación de muestras e instrumentos de laboratorio.

\section{MATERIALES Y MÉTODOS}

Para este trabajo de utilizó la ley de propagación de incertidumbre, que a su vez se sustenta en el desarrollo en serie de Taylor de una función compuesta, de manera a obtener la incertidumbre estándar compuesta, de acuerdo a la guía para la expresión de 
incertidumbres (ISO, 1995). Esta incertidumbre es la incertidumbre estándar del resultado de una medición indirecta, es decir que se utiliza cuando el resultado buscado, el cual no puede medirse directamente, es obtenido a partir de la medición de un conjunto de magnitudes directamente medibles. De acuerdo a lo anterior, cuando se desea conocer la incertidumbre estándar compuesta $u(y)$ de una magnitud $y$ que depende de otras magnitudes según una relación de tipo $y=f\left(x_{1}, x_{2}, \ldots, x_{\mathrm{N}}\right.$ ), se puede utilizar (1) (NF ENV 13005, 1999):

$u^{2}(y)=\sum_{i=1}^{N}\left(\frac{\partial f}{\partial x_{i}}\right)^{2} u^{2}\left(x_{i}\right)+2 \sum_{i=1}^{N-1} \sum_{j=i+1}^{N} \frac{\partial f}{\partial x_{i}} \frac{\partial f}{\partial x_{j}} u\left(x_{i}, x_{j}\right)$

donde $u\left(x_{\mathrm{i}}\right)$ es la incertidumbre estándar asociada a $x_{\mathrm{i}}$ y $u\left(x_{\mathrm{i}}, x_{\mathrm{j}}\right)$ es la covarianza estimada entre $x_{\mathrm{i}}$ y $x_{\mathrm{j}}$. Si se considera que todas las cantidades $x_{\mathrm{i}}$ y $x_{\mathrm{j}}$ se obtienen de forma independiente, las covarianzas $u\left(x_{\mathrm{i}}, x_{\mathrm{j}}\right)$ se pueden ignorar y por lo tanto (1) se simplifica de la siguiente manera para obtener (2):

$\mathrm{u}^{2}(\mathrm{y})=\sum_{\mathrm{i}=1}^{\mathrm{N}}\left(\frac{\partial \mathrm{f}}{\partial \mathrm{x}_{\mathrm{i}}}\right)^{2} \mathrm{u}^{2}\left(\mathrm{x}_{\mathrm{i}}\right)$

Con el objetivo de comparar dos resultados $x_{1}$ y $x_{2}$ de los que se conoce su incertidumbre $u\left(x_{1}\right)$ y $u\left(x_{2}\right)$, se utilizó la prueba t (3) (Ruxton, 2006):

$\mathrm{t}=\frac{\mathrm{x}_{1}-\mathrm{x}_{2}}{\sqrt{\frac{\mathrm{u}^{2}\left(\mathrm{x}_{1}\right)}{\mathrm{n}_{1}}+\frac{\mathrm{u}^{2}\left(\mathrm{x}_{2}\right)}{\mathrm{n}_{2}}}}$

donde $n_{1}$ y $n_{2}$ es el número de mediciones realizadas para obtener $x_{1}$ y $x_{2}$, respectivamente. Con ese parámetro $t$ calculado se procede a consultar las tablas estadísticas para la distribución normal con los grados de libertad $g l$ (estimador necesario para el cálculo de un estadístico particular, e indica el número de valores aleatorios que no pueden ser determinados o fijados mediante una ecuación matemática) (4): 
$\mathrm{gl}=\frac{\left(\frac{\mathrm{u}^{2}\left(\mathrm{x}_{1}\right)}{\mathrm{n}_{1}}+\frac{\mathrm{u}^{2}\left(\mathrm{x}_{2}\right)}{\mathrm{n}_{2}}\right)^{2}}{\frac{\left(\frac{\mathrm{u}^{2}\left(\mathrm{x}_{1}\right)}{\mathrm{n}_{1}}\right)^{2}}{\mathrm{n}_{1}-1}+\frac{\left(\frac{\mathrm{u}^{2}\left(\mathrm{x}_{2}\right)}{\mathrm{n}_{2}}\right)^{2}}{\mathrm{n}_{2}-1}}$

Adicionalmente, y como se explicará más adelante, se utilizó el método de Monte Carlo para el cálculo de incertidumbres teniendo en cuenta múltiples réplicas de cada ensayo por muestra. A manera de ejemplo de aplicación de los métodos de incertidumbre propuestos, éstos fueron aplicados a resultados de concentración en Sólidos Suspendidos Totales (SST), obtenidos según la norma 2540D de Standard Methods for the Examination of Water and Wastewater (1998), relativas a muestras de agua suministradas por el Laboratorio de Pruebas y Ensayos de la Facultad de Ingeniería de la Pontificia Universidad Javeriana.

\section{METODOLOGÍA}

Una vez recibida la muestra (de aproximadamente $1 \mathrm{~L}$ ) en el laboratorio, se procede a estimar su concentración del parámetro escogido, según las necesidades del estudio (SST, DQO, DBO 5 , etc.) Esta estimación requiere que se realice un submuestreo, en virtud a que la totalidad de la muestra recolectada en el cuerpo receptor no puede ser utilizada para el establecimiento de las concentraciones. Por ejemplo, para determinar la concentración en SST, es usual tomar un volumen entre $20 \mathrm{~mL}$ y $100 \mathrm{~mL}$, dependiendo de la concentración de la muestra (para evitar posibles colmataciones de los poros del filtro utilizado durante el ensayo).

En algunas ocasiones, para estimar el resultado de concentración de una submuestra, es necesario utilizar diferentes instrumentos de medición y/o diferentes procedimientos de laboratorio. Generalmente, la muestra es sometida a una cadena de mediciones. Debido a lo anterior, la incertidumbre asociada al resultado de medición no depende únicamente de la precisión de un sólo aparato. Es necesario entonces recurrir a la ley de propagación de 
incertidumbres, la cual se presentó en la Sección 2. Por ejemplo, para la obtención del resultado de concentración en SST, se debe medir el peso seco de un filtro con poros de 0,45 $\mu \mathrm{m} M_{\mathrm{i}}$, el peso seco del filtro más los sólidos retenidos en dicho filtro $M_{\mathrm{f}}$ y el volumen de la submuestra $V$. El resultado de concentración $S S T_{\mathrm{i}}$ asociado a la submuestra $i$ de volumen $V$ se calcula de acuerdo a (5):

$\operatorname{SST}_{\mathrm{i}}=\frac{\mathrm{M}_{\mathrm{f}}-\mathrm{M}_{\mathrm{i}}}{\mathrm{V}}$

De manera a obtener la incertidumbre $u\left(S S T_{\mathrm{i}}\right)$ asociada al re-

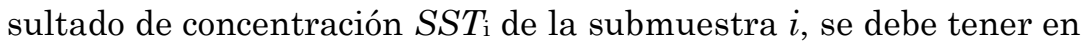
cuenta la precisión $p_{\mathrm{m}}$ de la balanza que sirve para medir las masas $M_{\mathrm{i}}$ y $M_{\mathrm{f}}$, así como la precisión $p \mathrm{v}$ de la probeta que sirve para medir el volumen $V$ de la submuestra $i$. Si se considera que las mediciones de las masas y del volumen con dichos aparatos siguen una distribución normal, se puede asumir que las incertidumbres $u\left(M_{\mathrm{i}}\right), u\left(M_{\mathrm{f}}\right)$ y $u(V)$ asociadas a la medición de $M_{\mathrm{i}}, M_{\mathrm{f}} \mathrm{y} V$, utilizando dichos aparatos, son iguales a la mitad de $p_{\mathrm{m}} \mathrm{y}$ de $p_{\mathrm{v}}$, respectivamente, con un $95 \%$ de confianza. Adicionalmente, si se asume que las mediciones de $M_{\mathrm{i}}, M_{\mathrm{f}}$ y $V$ son independientes, se puede utilizar (2) para el cálculo de la incertidumbre estándar compuesta. Para este caso específico, la ecuación toma la forma de (6):

$\mathrm{u}\left(\operatorname{SST}_{\mathrm{i}}\right)=\frac{1}{\mathrm{~V}} \sqrt{\mathrm{u}^{2}\left(\mathrm{M}_{\mathrm{i}}\right)+\mathrm{u}^{2}\left(\mathrm{M}_{\mathrm{f}}\right)+\operatorname{SST}_{\mathrm{i}}^{2} \mathrm{u}^{2}(\mathrm{~V})}$

El cálculo de la incertidumbre asociada a cada réplica $i$ es útil tanto para estimar la incertidumbre estándar compuesta como para depurar e interpretar los resultados en bruto obtenidos en laboratorio. Sobre este último aspecto, la metodología propuesta contempla una comparación de los resultados de cada réplica teniendo en cuenta sus incertidumbres estándar asociadas. Si se asume que los resultados de las mediciones de cada réplica siguen una distribución normal, se propone una comparación por pares mediante pruebas t, utilizando (3) y (4). De acuerdo a los resultados de dichas pruebas, pueden existir tres posibilidades: (i) no se elimina ninguna réplica, al no detectar diferencias significativas 
(valores $p$ entre parejas superiores a 0,05 ); (ii) no se elimina ninguna réplica porque se detectan diferencias significativas entre todas las réplicas (valores $p$ entre parejas inferiores a 0,05); (iii) se elimina una sola réplica porque se detectan diferencias significativas entre una réplica y las dos restantes.

Una vez tomada la decisión sobre la aceptación o el rechazo de cada réplica, se propone el cálculo de la incertidumbre asociada a la muestra, considerando tanto la variabilidad entre los resultados de las réplicas como la incertidumbre de cada réplica considerada. El cálculo propuesto, explicado en seguida, se basa en el método de Monte Carlo: Teniendo en cuenta el valor medido y la incertidumbre asociada a la réplica 1 de la muestra $j$, se escoge aleatoriamente un valor de prueba $x_{1, \mathrm{j}}$ de acuerdo con una distribución normal asumiendo el valor medido como el promedio en la distribución y la incertidumbre asociada como su desviación estándar. De manera similar se escogen valores de prueba $x_{2, j}$ y $x_{3, j}$ para las réplicas número 2 y 3 , respectivamente. Para esos valores $x_{1, \mathrm{j}}, x_{2, \mathrm{j}}$ y $x_{3, \mathrm{j}}$, se calcula la desviación estándar correspondiente $\sigma_{j}$. Posteriormente, se seleccionan aleatoriamente nuevos valores de prueba $x_{1, j+1}, x_{2, j+1}$ y $x_{3, j+1}$, de tal forma que se pueda calcular la desviación estándar entre esos resultados $\sigma_{j+1}$ correspondientes a la iteración $j+1$. Al cabo de un número elevado $n$ de iteraciones, se tienen $n$ valores de desviaciones estándar entre las réplicas $\left(\sigma_{j}, \sigma_{j+1}, \ldots, \sigma_{j+n-1}\right)$. El promedio de dichas desviaciones estándar se interpreta como la incertidumbre compuesta más probable $u_{\mathrm{cj}}$ asociada a la muestra $j$.

La metodología presentada arriba fue la base para el desarrollo del programa CIMA (Cálculo de Incertidumbres asociadas a datos de calidad de aguas mediante Métodos de generación Aleatoria) utilizando el paquete estadístico $R$ ( $R$ Development Core Team, 2011).

\section{RESULTADOS Y DISCUSIÓN}

El Laboratorio de Pruebas y Ensayos de la Facultad de Ingeniería de la Pontificia Universidad Javeriana (PUJ) suministró los siguientes resultados correspondientes a tres muestras de agua tomadas en diferentes fuentes, con el fin de estimar la respectiva 
concentración en SST (ver Tabla 1). Como se puede observar en la Tabla 1, los resultados seleccionados corresponden a muestras reales con bajas, medianas y elevadas concentraciones de SST y con diferencias en las variabilidades submuestrales. Mediante el método de propagación de incertidumbres utilizando (6), se estimó la incertidumbre asociada a cada réplica, teniendo en cuenta que las precisiones de la balanza y de la probeta utilizadas fueron de $0,1 \mathrm{mg}$ y $0,03 \mathrm{~mL}$, respectivamente. Asumiendo que al medir repetidamente con dichos aparatos los resultados obtenidos siguen una distribución normal, se estimó que las incertidumbres asociadas a cada medición eran de 0,05 mg y de $0,015 \mathrm{~mL}$, para la balanza y para la probeta respectivamente.

Tabla 1. Concentración de sólidos suspendidos totales para tres muestras de agua (Muestra 1: tomada el 3/11/2009 en la cuenca media del Río Arzobispo; Muestra 2: tomada el 8/04/2010 en el canal de aguas residuales del campus de la PUJ; Muestra 3: tomada durante la lluvia del 6/04/2010 en la cubierta del edificio de parqueaderos del campus de la PUJ)

\begin{tabular}{ccccccc}
\hline Muestra & Réplica & Código & $M_{\mathrm{i}}(\mathrm{g})$ & $M_{\mathrm{f}}(\mathrm{g})$ & $V(\mathrm{~mL})$ & $\mathrm{SST}(\mathrm{mg} / \mathrm{L})$ \\
\hline \multirow{2}{*}{1} & 1 & $\mathrm{~m}_{11}$ & 21,4323 & 21,4375 & 30 & 173 \\
& 2 & $\mathrm{~m}_{12}$ & 21,6154 & 21,6205 & 30 & 170 \\
& 3 & $\mathrm{~m}_{13}$ & 21,5780 & 21,5823 & 25 & 172 \\
2 & 1 & $\mathrm{~m}_{21}$ & 18,4732 & 18,4860 & 20 & 640 \\
& 2 & $\mathrm{~m}_{22}$ & 24,8534 & 24,8644 & 20 & 550 \\
& 3 & $\mathrm{~m}_{23}$ & 21,3943 & 21,4065 & 20 & 610 \\
3 & 1 & $\mathrm{~m}_{31}$ & 21,3945 & 21,3965 & 50 & 40 \\
& 2 & $\mathrm{~m}_{32}$ & 23,9829 & 23,9856 & 50 & 54 \\
& 3 & $\mathrm{~m}_{33}$ & 17,9873 & 17,9901 & 50 & 56 \\
\hline
\end{tabular}

$M_{\mathrm{i}}$ : peso seco de un filtro con poros de $0,45 \mu \mathrm{m}$

$M_{\mathrm{f}:}$ peso seco del filtro más los sólidos retenidos en dicho filtro

$V$ : volumen de la submuestra

SST: concentración de Sólidos Suspendidos Totales de la submuestra

Aplicando (6) a los resultados presentados en la Tabla 1, se obtuvieron los resultados de incertidumbre asociados a cada réplica mostrados en la Tabla 2. 
Tabla 2. Resultados de incertidumbre para SST asociada a cada réplica

\begin{tabular}{cccccc}
\hline Muestra & Réplica & Código & SST $(\mathrm{mg} / \mathrm{L})$ & $\begin{array}{c}u(\mathrm{SST}) \\
(\mathrm{m} / \mathrm{L})\end{array}$ & $u(\mathrm{SST})(\%)$ \\
\hline \multirow{2}{*}{1} & 1 & $\mathrm{~m}_{11}$ & 173 & 2,358615 & 1,36 \\
& 2 & $\mathrm{~m}_{12}$ & 170 & 2,358555 & 1,39 \\
& 3 & $\mathrm{~m}_{13}$ & 172 & 2,830309 & 1,65 \\
2 & 1 & $\mathrm{~m}_{21}$ & 640 & 3,567969 & 0,56 \\
& 2 & $\mathrm{~m}_{22}$ & 550 & 3,559516 & 0,65 \\
& 3 & $\mathrm{~m}_{23}$ & 610 & 3,565011 & 0,58 \\
3 & 1 & $\mathrm{~m}_{31}$ & 40 & 1,414264 & 3,54 \\
& 2 & $\mathrm{~m}_{32}$ & 54 & 1,414306 & 2,62 \\
& 3 & $\mathrm{~m}_{33}$ & 56 & 1,414313 & 2,53 \\
\hline
\end{tabular}

SST: concentración de Sólidos Suspendidos Totales de la submuestra u(SST): incertidumbre sobre el valor de SST obtenido

Teniendo en cuenta las incertidumbres presentadas en la tabla anterior, las Fig. 1 a Fig. 3 representan respectivamente la variabilidad de cada réplica para las muestras 1 a 3 de la Tabla 1 . El rango mostrado corresponde al rango más probable (probabilidad de 95\%) de variación de los valores de SST para cada réplica.

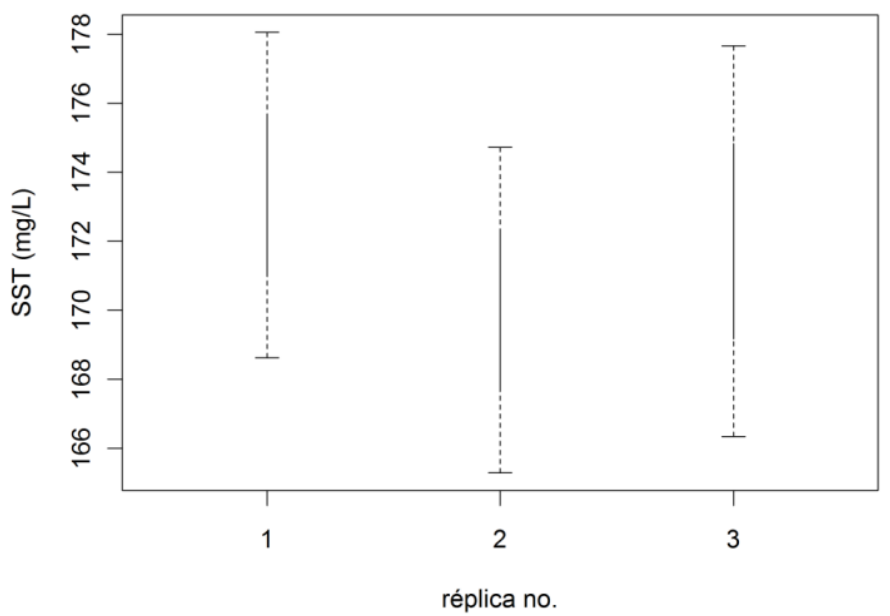

Fig. 1. Variabilidad de cada réplica de la muestra 1 


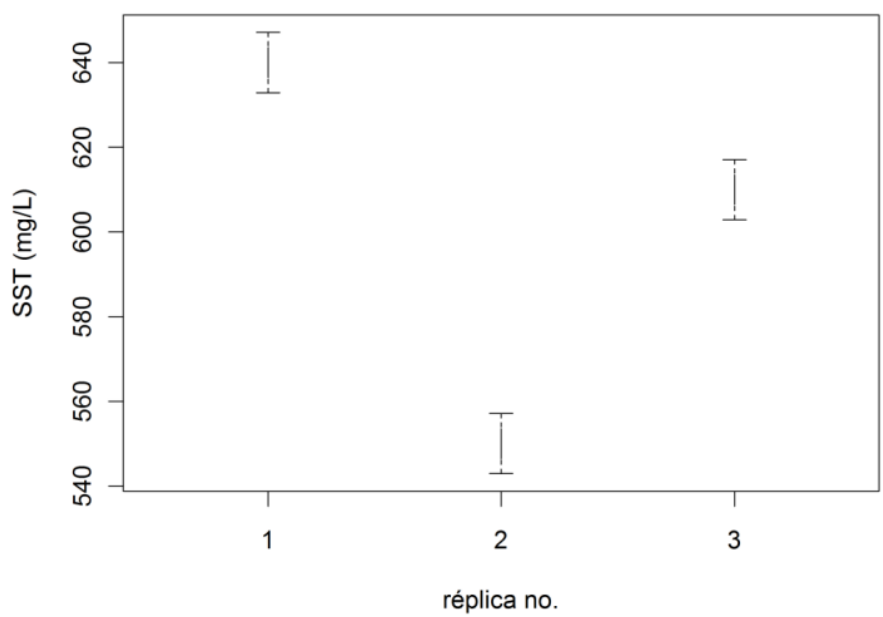

Fig. 2. Variabilidad de cada réplica de la muestra 2

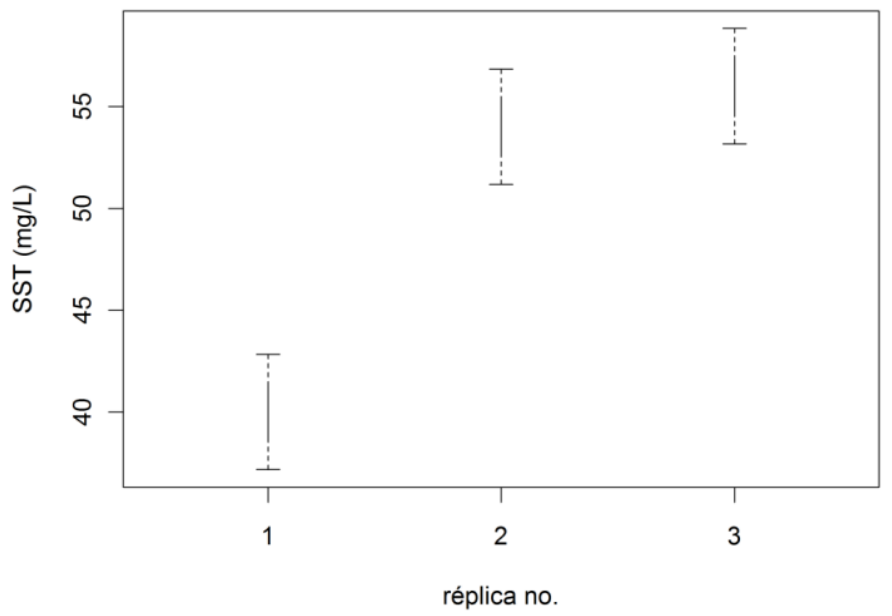

Fig. 3. Variabilidad de cada réplica de la muestra 3

En las Tablas 1 y 2 y en las Fig. 1 a 3 se observa que podría existir una alta variabilidad entre réplicas de una misma muestra, y por lo tanto el resultado de una única réplica podría no ser representativo de la concentración real de un contaminante en la 
muestra. En la Tabla 3 se presentan los valores promedio para cada muestra, considerando las tres réplicas en cada caso. Si se toma una única réplica por muestra, se podría estar cometiendo un error hasta del $20 \%$ con respecto al valor promedio de la muestra (caso de la réplica $\mathrm{m}_{31}$ Tabla 2 ). Si se tienen en cuenta todas las réplicas de cada muestra, se podría calcular la incertidumbre con base únicamente en la precisión de los aparatos de medición empleados.

Tabla 3. Concentraciones promedio de SST e incertidumbres asociadas considerando únicamente la precisión de los instrumentos de laboratorio empleados (sin considerar la variabilidad de los resultados entre réplicas)

\begin{tabular}{cccc}
\hline Muestra & SST $(\mathrm{mg} / \mathrm{L})$ & $u(\mathrm{SST})(\mathrm{mg} / \mathrm{L})$ & $u(\mathrm{SST})(\%)$ \\
\hline 1 & 171,78 & 1,46 & 0,85 \\
2 & 600,00 & 2,06 & 0,34 \\
3 & 50,00 & 0,82 & 1,63 \\
\hline
\end{tabular}

Bajo esta premisa, basta calcular la incertidumbre asociada al valor promedio de cada muestra por medio de la ley de propagación de la incertidumbre (ver resultados Tabla 3). Como se observa en la Tabla 3, la incertidumbre calculada de esta manera resulta muy pequeña (inferior a $2 \%$ sobre el valor promedio), ya que no se tiene en cuenta la variabilidad de los resultados entre réplicas. Este método resulta entonces poco conveniente cuando se quiere asociar el resultado de incertidumbre al valor promedio de concentración en una muestra en presencia de una alta variabilidad de los resultados de las réplicas, lo cual se quiso subsanar mediante la propuesta metodológica propuesta, cuyos resultados se presentan en los siguientes párrafos.

Sobre la base de las pruebas t (3) y (4) se estableció que: (i) para la muestra 1 , todas las réplicas son similares, es decir que no existen diferencias significativas entre ellos (valores $p>0,05$ ) y por lo tanto se tienen que conservar todas las réplicas para los cálculos posteriores; (ii) para la muestra 2, todas las réplicas presentan diferencias significativas (valores $p<0,05$ ) y por lo tanto se tienen que conservar todas las réplicas para los cálculos posteriores; (iii) para la muestra 3, la réplica 1 presenta diferencias significativas con respecto a las otras dos réplicas (valores $p$ de 0,00027 
y 0,00016$)$ mientras que las réplicas 2 y 3 no presentan diferencias significativas (valor $p$ de 0,1583 ); por lo tanto se decide eliminar la réplica número 1 para los cálculos posteriores.

De acuerdo con los resultados antes mencionados, se conservan todas las réplicas de las muestras 1 y 2 , mientras que para la muestra 3 se conservan únicamente las réplicas 2 y 3 . Posteriormente se procede a hacer la simulación de Monte Carlo. En las Fig. 4 a 6 se representan las secuencias de generación de 5000 ternas de réplicas generadas aleatoriamente para las muestras $1 \mathrm{a}$ 3 , respectivamente. Para propósitos ilustrativos se muestra la selección aleatoria de 10,50,100, .., 5000 tripletas o parejas de réplicas, de acuerdo con los resultados obtenidos al aplicar el método de eliminación basado en pruebas t. Al cabo de las 5000 generaciones propuestas, se logró estimar la incertidumbre compuesta asociada al resultado promedio de concentración de cada muestra.
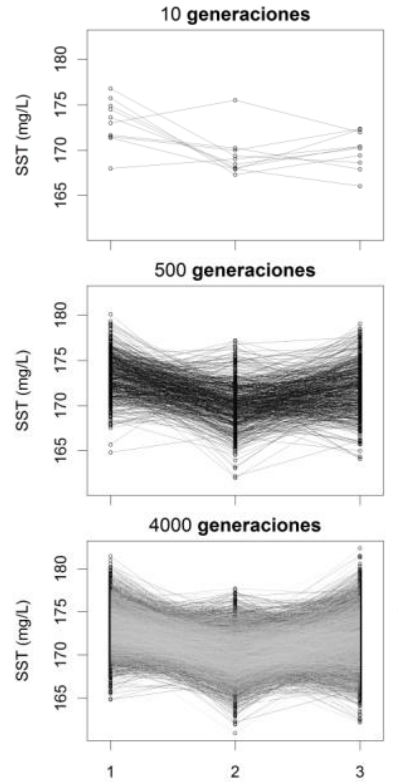

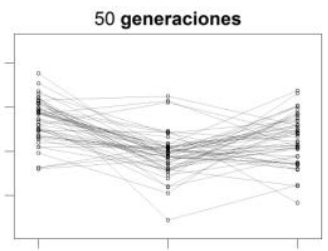

1000 generaciones

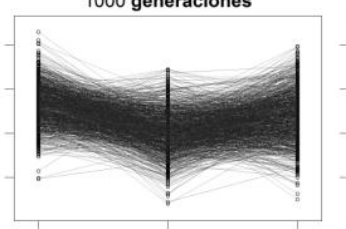

4545 generaciones

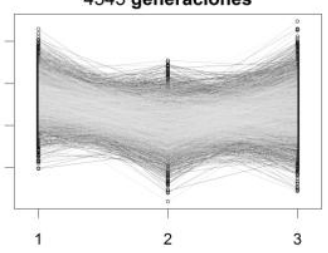

100 generaciones

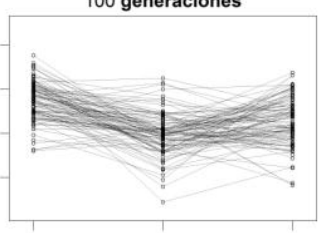

2500 generaciones

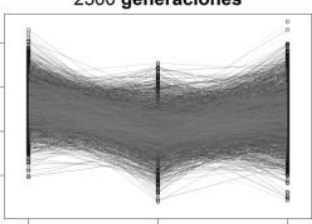

5000 generaciones

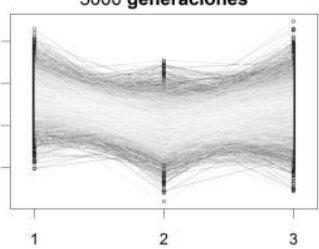

Fig. 4. Simulación de Monte Carlo de 5000 ternas de réplicas para la muestra 1 

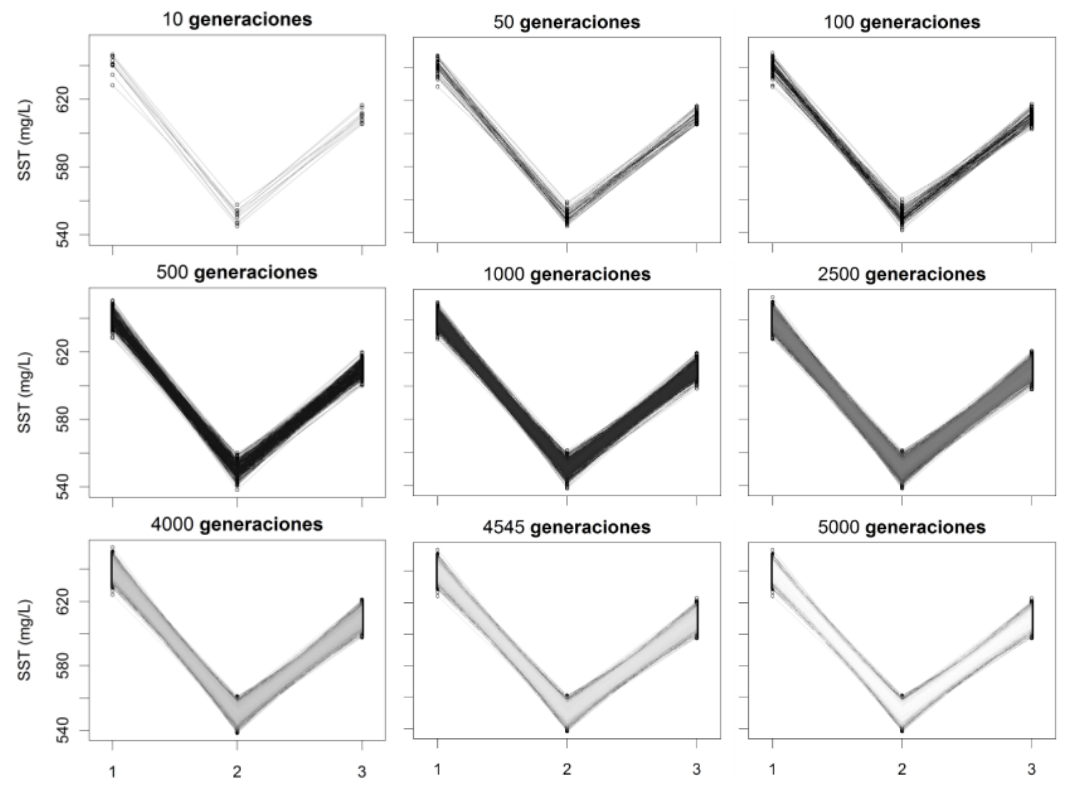

Fig. 5. Simulación de Monte Carlo de 5000 ternas de réplicas para la muestra 2
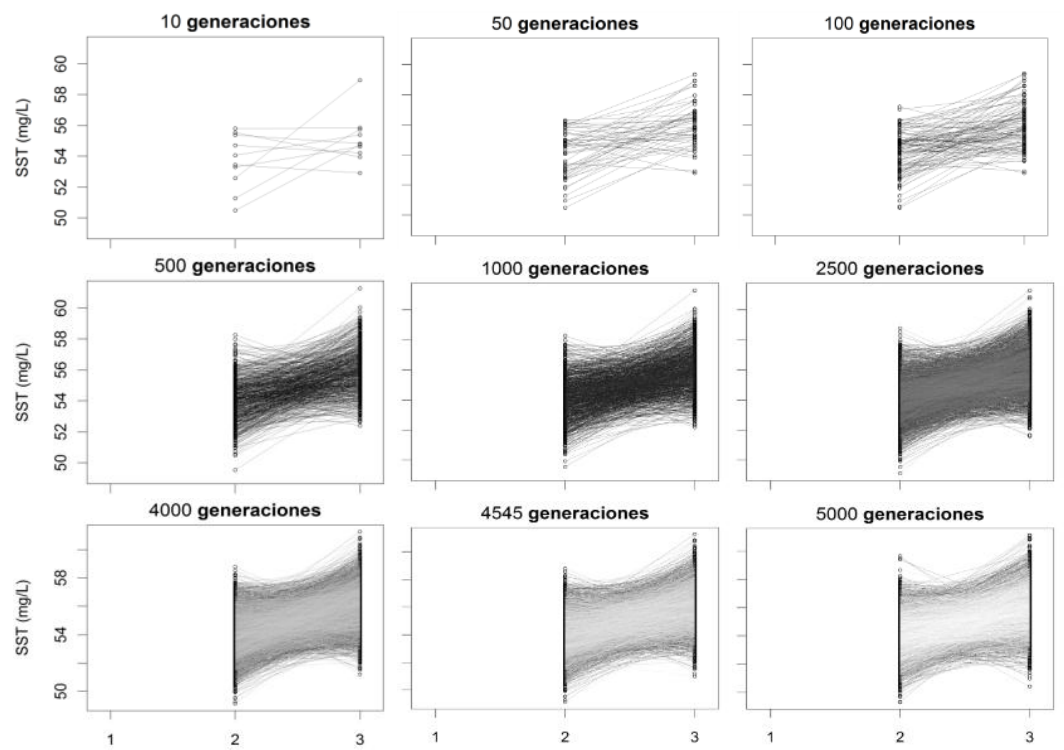

Fig. 6. Simulación de Monte Carlo de 5000 ternas de réplicas para la muestra 3 
Los resultados de concentración promedio e incertidumbre asociada obtenidas al aplicar el programa CIMA (el cual incluye la ley de propagación de incertidumbres aplicada al resultado de cada réplica, el método de eliminación de réplicas mediante pruebas t y el método de Monte Carlo) se muestran en la Tabla 4. Considerando los resultados de concentración de SST para cada muestra presentados en Tabla 4 y comparándolos con los valores de concentración de SST obtenidos para cada réplica presentados en Tablas 1 y 2 , se observan diferencias hasta de $27 \%$ entre el valor de cada réplica y el valor promedio de la muestra correspondiente (caso de la réplica $\mathrm{m}_{31}$ Tabla 2 ).

Tabla 4. Concentraciones promedio de SST e incertidumbres asociadas calculadas utilizando el programa CIMA

\begin{tabular}{cccc}
\hline Muestra & SST $(\mathrm{mg} / \mathrm{L})$ & $u(\mathrm{SST})(\mathrm{mg} / \mathrm{L})$ & $u(\mathrm{SST})(\%)$ \\
\hline 1 & 171,78 & 2,70 & 1,57 \\
2 & 600,00 & 45,84 & 7,64 \\
3 & 55,00 & 1,65 & 3,00 \\
\hline
\end{tabular}

Comparando los resultados presentados en Tabla 4 (obtenidos utilizando la metodología propuesta mediante el programa CIMA) con aquellos presentados en Tabla 3 (obtenidos con base únicamente en la precisión de los aparatos de medición empleados, mediante la ley de propagación de incertidumbres), se observa que aunque los resultados de concentración promedio para las muestras 1 y 2 no cambiaron, para la muestra 3 la concentración promedio es $10 \%$ mayor utilizando el método propuesto con respecto al valor de concentración presentado en Tabla 3, debido a que se eliminó la réplica número 1. Esta diferencia entre los valores promedio de SST, sin tener en cuenta las incertidumbres asociadas, podría traer consecuencias importantes en la toma de decisiones en la gestión de los hidrosistemas asociados, ya que por ejemplo, en un caso dicho valor podría estar por debajo de algún valor límite normativo, mientras que en otro caso no. Adicionalmente, los resultados de las incertidumbres obtenidas utilizando el método propuesto son mucho mayores que los presentados en Tabla 3 (diferencias de más del 85\%). 
La Fig. 7 proporciona una comparación de las incertidumbres relativas (en valor porcentual) para las tres muestras estudiadas calculadas utilizando, por un lado el método ordinario, por medio únicamente de la precisión de los instrumentos de medición empleados en laboratorio, mediante la ley de propagación de la incertidumbre, y por otro lado utilizando el método propuesto a través del programa CIMA.

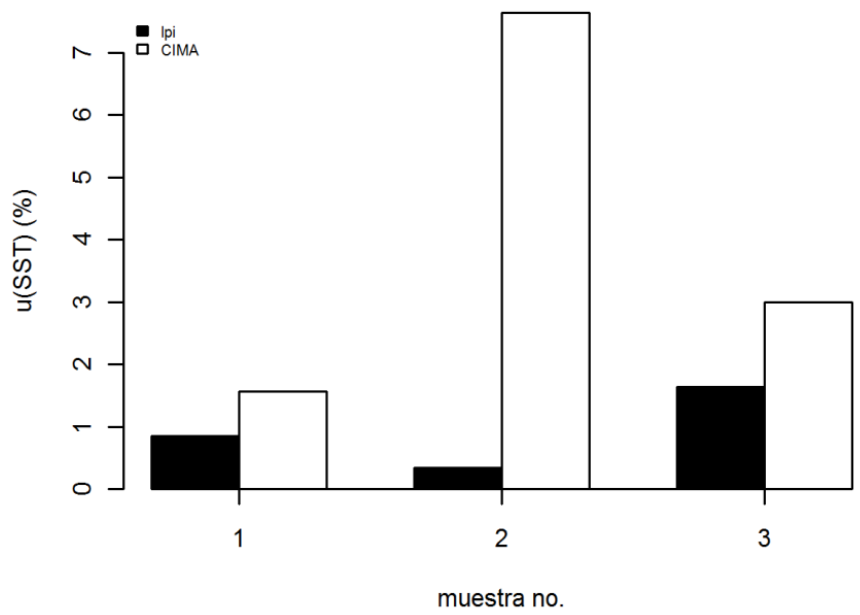

Fig. 7. Comparación de incertidumbres obtenidas contemplando únicamente la precisión de los instrumentos de laboratorio, mediante la ley de propagación de incertidumbres (etiqueta "lpi") y el programa CIMA (etiqueta "CIMA")

\section{CONCLUSIONES}

Este trabajo presenta una propuesta metodológica para la estimación de incertidumbres asociadas a resultados de concentraciones de Sólidos Suspendidos Totales (SST) sobre muestras puntuales obtenidas en campo, contemplando no solamente la precisión de los instrumentos de medición en laboratorio sino también la variabilidad presente en el submuestreo y la manipulación de muestras y aparatos en laboratorio.

La metodología desarrollada contempla: (i) la estimación de incertidumbres asociadas a réplicas mediante la ley de propagación 
de incertidumbres; (ii) pruebas t para comparar los resultados asociados a réplicas, teniendo en cuenta la variabilidad de cada una de ellas, y poder tomar decisiones sobre la aceptación o el rechazo de los resultados de réplicas para una misma muestra; (iii) el método de Monte Carlo para estimar la incertidumbre compuesta asociada al resultado de concentración de contaminante para una muestra. Esta metodología fue el objeto del programa CIMA, desarrollado en el paquete estadístico $\mathrm{R}$ ( $\mathrm{R}$ Development Core Team, 2011).

Mediante un ejemplo de aplicación, se logró comprobar que la incertidumbre evaluada con el método propuesto puede llegar a ser mayor que aquella obtenida mediante métodos más tradicionales, los cuales contemplan únicamente la precisión de los instrumentos de medición utilizados, por medio de métodos analíticos. Además, se observa que los resultados de concentración asociados a una muestra pueden variar al aplicar la metodología propuesta, en comparación con los resultados promedios obtenidos aplicando metodologías más tradicionales. Esto puede traer consecuencias importantes a la hora de tomar decisiones asociadas a la gestión de fuentes de agua ya que, por ejemplo, en un caso, el valor de concentración podría estar por debajo de algún valor límite normativo, mientras que en otro caso no. Así mismo, si se utilizan los resultados promedio obtenidos, sin contemplar incertidumbres, para el cálculo de cargas contaminantes o para simulaciones hidrodinámicas durante periodos prolongados (periodos anuales o multianuales) de funcionamiento del hidrosistema asociado, a término podrían presentarse diferencias mucho más importantes, y en algunos casos dichas evaluaciones soportarían de forma inadecuada la toma de decisiones para la cual se realizaron.

\section{REFERENCIAS}

CEM, Centro Español de Metrología, (2008); Vocabulario internacional de metrología - Conceptos fundamentales y generales, y términos asociados (VIM), $3^{\text {a }}$ edición, Madrid, España, 88p.

EURACHEM/CITAC Guide, (2000); Quantifying uncertainty in analytical measurement, Second Edition, Editors: S L R Ellison (LGC, UK) M Rosslein (EMPA, Switzerland) A Williams (UK). 126p. 
Ghestem, J.P., Lachenal, J., (2008); Incertitude sur l'échantillonnage et le prélèvement d'eaux : synthèse bibliographique, BRGM/RP-56885-FR, $58 \mathrm{p}$.

Greenland, S., (2001); Sensitivity analysis, Monte Carlo risk analysis, and Bayesian uncertainty assessment, Risk Anal, 21(4), 579-583.

Harmel, R.D., Smith, D.R., King, K.W., Slade, R.M., (2009); Estimating storm discharge and water quality data uncertainty: A software tool for monitoring and modeling applications, Environmental Modelling and Software, 24(7), 832-842.

ISO, International Organization for Standardization, (1995); Guide to the expression of uncertainty in measurement, Geneva: ISO, 1995: 101p.

Isukapalli, S.S., Georgopoulos, P.G., (2001); Computational methods for sensitivity and uncertainty analysis for environmental and biological models (EPA/600/R-01-068), Research Triangle Park, NC. US EPA, National Exposure Research Laboratory, 145p.

JCGM, Joint Committee for Guides in Metrology, (2008); International vocabulary of metrology: basic and general concepts and associated terms (VIM). Sèvres, France: JCGM, 2008. Available from: http://www.bipm.org/en/publications/guides/vim.html.

Ministerio de Desarrollo Económico, (2000); Reglamento Técnico del Sector de Agua Potable y Saneamiento Básico, RAS - 2000. Sección II, Título E Tratamiento de Aguas Residuales, República de Colombia.

Ministerio de la Protección Social, Ministerio de Ambiente Vivienda y Desarrollo Territorial, (2007); Por medio de la cual se señalan características, instrumentos básicos y frecuencias del sistema de control y vigilancia para la calidad del agua para consumo humano, Resolución 2115 de 2007, Bogotá, D.C.

Ministerio de Salud, (1984); Decreto 1594, Usos del agua y residuos líquidos, República de Colombia, 52p.

NF ENV 13005, (1999); Guide pour l'expression de l'incertitude de mesure, Paris (France), AFNOR, août, 113p.

Poulter, S.R., (1998); Monte Carlo simulation in environmental risk assessment - science, policy and legal issues, Risk: Health, Safety \& Environment, 9, 7-26.

R Development Core Team, (2011); R: A language and environment for statistical computing, R Foundation for Statistical Computing, Vienna, Austria, URL http://www.R-project.org/. 
Radwan, M., Willems, P., (2008); Uncertainty analysis for river quality measurements, International Journal of Ecological Economics \& Statistics (IJEES), 11(S08), 21-30.

Ruxton, G.D., (2006); The unequal variance t-test is an underused alternative to Student's t-test and the Mann-Whitney U test, Behavioral Ecology, 17(4), 688-690.

Smith, E., (2002); Uncertainty analysis, In: Encyclopedia of Environmetrics, Wiley, 4, 2283-2297.

Standard Methods for the Examination of Water and Wastewater, (1998); 20th edn, American Public Health Association/American Water Works Association/Water Environment Federation, Washington DC, USA. 1325 .

Taverniers, I., De Loose, M., Van Bockstaele, E., (2004a); Trends in quality in the analytical laboratory. I. Traceability and measurement uncertainty of analytical results, Trends Anal. Chem., 23(7), 480-490.

Taverniers, I., De Loose, M., Van Bockstaele, E., (2004b); Trends in quality in the analytical laboratory. II. Analytical method validation and quality assurance, Trends Anal. Chem., 23(8), 535-552. 